\title{
Essais
}

ESSAIS

Revue interdisciplinaire d'Humanités

Hors-série 6 | 2021

Agrobiodiversité et territoires

\section{Le Porc Noir Gascon : élever et valoriser une race locale rustique en situation péri-urbaine de Bordeaux}

Rencontre avec Isabelle Ortusi

The Gascon Black Pig: raising and developing a rustic local breed in the suburbs of Bordeaux

Meeting with Isabelle Ortusi

\section{OpenEdition}

\section{Édition électronique}

URL : https://journals.openedition.org/essais/7961

DOI : 10.4000/essais.7961

ISSN : 2276-0970

Éditeur

École doctorale Montaigne Humanités

Édition imprimée

Date de publication : 1 mars 2021

Pagination : 187-190

ISBN : 978-2-492780-00-4

ISSN : 2417-4211

Référence électronique

"Le Porc Noir Gascon : élever et valoriser une race locale rustique en situation péri-urbaine de Bordeaux », Essais [En ligne], Hors-série 6 | 2021, mis en ligne le 16 mars 2021, consulté le 18 janvier 2023. URL : http://journals.openedition.org/essais/7961 ; DOI : https://doi.org/10.4000/essais.7961 


\section{Le Porc Noir Gascon : élever et valoriser une race locale rustique en situation péri-urbaine de Bordeaux}

\section{Entretien avec Isabelle Ortusi ${ }^{1}$}

Isabelle Ortusi est exploitante agricole et éleveuse, co-fondatrice du Domaine de Jarry à Cestas.

\section{Pouvez-vous nous présenter votre production, ainsi que le cadre paysager du domaine?}

Le domaine de Jarry est situé sur la commune de Cestas, au sud de Bordeaux, le long de l'autoroute A63 qui mène vers Bayonne. À vol d'oiseau, c'est à $1 \mathrm{~km}$ de l'autoroute, dans une zone très agricole, de plaines céréalières à production de légumes, mais aussi forestière avec de grands îlots de sylviculture de pins maritimes. Avec la proximité de l'autoroute, de grandes plateformes de logistiques ou zones d'activités se sont développées. La densité de plateformes logistiques a amené de l'emploi dans la région, menant Cestas et les communes alentours à se développer en grandes zones d'habitations et de lotissements, en relation avec le développement de la métropole bordelaise et du bassin d'Arcachon. Il y a 20 ans, chez nous, c'était très reculé alors que maintenant ce n'est plus le cas, nous sommes en territoire périurbain.

Le domaine est au cour de ce que l'on appelle une lande girondine; historiquement, c'était des terrains marécageux, très humides. La planéité du relief fait qu'il y avait très peu de drainage des eaux, si bien que jusqu'au XIX ${ }^{e}$ siècle, c'était des zones considérées comme insalubres. Au cours du XIXe siècle, il y a eu une énorme transformation du territoire, avec la plantation massive de forêts de pin maritime, constituant aujourd'hui l'une des forêts de monoculture les plus grandes d'Europe. Tout cela sous un climat océanique et sur un sol sableux, très pauvre, sans intérêt majeur.

1 Dans le cadre de son intervention au séminaire les 14 et 15 mai 2019. 


\section{Comment vous êtes vous implantés sur le domaine?}

On a racheté ce domaine, qui fait à peu près 50 hectares, à la fin des années 1990 ; c'était alors un reste de coupe rase après exploitation des pins, où l'on souhaitait régénérer un biotope. Au départ, il y avait un peu de repousses de feuillus avec des châtaigniers, chênes, robiniers, érables, quelques figuiers et pruniers sauvages, et de la végétation broussailleuse de lande avec des bruyères, genêts et brandes. Nous avons protégé tous ces arbres consciencieusement au fil des années, et nous avons replanté dans des zones où il n'y avait pas de repousses naturelles, afin de recréer une forêt. Nous avons implanté aussi quelques hectares de prairie permanente, dans l'idée d'y mettre les moutons ; mais avec les phases de sècheresse qui devenaient de plus en plus importantes, nous avons réimplanté des arbres sur ces espaces pour procurer de l'ombre aux animaux et surtout pour garder de l'herbe verte en plein été. C'est un des principes de base de l'agroforesterie.

\section{Quel est votre mode de production ? Quels liens faites-vous entre vos produits, le paysage et le territoire?}

Sur le domaine, nous avons mis en place trois élevages qui sont des ateliers complémentaires; nous voulions des animaux et c'était clair que nous allions les élever en plein air. Compte-tenu de la zone, nous voulions des races locales et rustiques, en capacité de valoriser un territoire pauvre, avec une végétation particulière. Nous nous sommes donc rapprochés du Conservatoire des Races d'Aquitaine pour choisir des poules gasconnes, des moutons landais et des porcs gascons. Les moutons landais et les porcs gascons étant par ailleurs des races à faibles effectifs, nous participons à la préservation de ces races-là en les élevant. L’objectif principal est de commercialiser des animaux vivants pour que des cheptels puissent se reconstituer un peu partout, en diffusant les races pour en augmenter les effectifs, même s'il y a toujours une part qui est valorisée en boucherie.

Pour le porc gascon, nous avons sur l'élevage 10 porcs, 8 truies et 2 verrats, cantonnés sur une zone de deux hectares elle-même divisée en 4 petits parcs de 0,5 hectares chacun. L'ensemble est clôturé pour des raisons sanitaires ; et entre chaque parc, nous avons recréé des buttes de terre de 2,50 m de haut plantées avec des arbustes et de l'herbe, où nous avons favorisé la repousse naturelle des feuillus. Cette haie sur butte a plusieurs objectifs : elle fait de l'ombre aux animaux en été, produit des aliments de compléments pour les cochons en automne (glands et figues), et limite l'érosion de la butte qui elle empêche les passages d'animaux d'un parc à l'autre. Cette organisation paysagère des parcs se retrouve à deux endroits, ce qui permet une rotation tous les 5 à 8 ans, quand le sol est trop appauvri par le passage des cochons. Changer de zone permet de faire baisser la charge parasitaire du sol qui vient au fil du temps ; et quand on sort les porcs d'un côté pour les mettre de l'autre, on laisse la végétation se régénérer, et ça devient une zone accessible aux moutons jusqu'à ce que l'on remette les cochons dans un autre cycle. 
Le cheptel de moutons est lui constitué de 130 brebis et 5 béliers, et ces bêtes sont des acteurs directs du paysage. Les animaux ont accès en pâturage à l'ensemble du domaine, forêts et prairies, dans des parcs fixes grillagés de taille variable en rotation permanente en fonction des disponibilités alimentaires. Le mouton est un agent gestionnaire direct, car il garde la végétation à une hauteur assez basse. Le vrai risque en forêt landaise, c'est l'incendie, et nous avons comme devoir d'entretenir nos parcelles de forêts pour limiter la propagation des feux ; avoir des moutons qui broutent nous épargne plusieurs dizaines d'heures de broyage mécanique coûteux par an. Les brebis valorisent une végétation de lande qui n'a pas de qualité nutritionnelle exceptionnelle ; et en tournant sur les parcelles, elles enrichissent le sol au fur et à mesure, permettant l'implantation d'une végétation de plus en plus diversifiée et de plus en plus dense. À noter que je ne traite mes animaux qu'en phytothérapie sauf cas exceptionnel, ce qui nous permet de protéger la pédofaune (déjections exempts de produits chimiques) et d'avoir un sol qui petit à petit devient quelque chose de riche, d'aéré.

Avoir favorisé les repousses des feuillus engendre également la recréation d'un humus qui participe à la désacidification et à la modification de la végétation, et donc, à fortiori, du paysage qui est moins uniforme.

Les poules gasconnes représentent un atelier plus petit sur l'exploitation. Elles sont réparties dans trois poulaillers distincts fermés par des grillages électrifiés pour limiter la prédation par les renards ; l'un d'eux ne possède pas de coqs pour permettre la production d'œufs commercialisables. Les deux autres sont destinées à produire des poussins pour renouveler le cheptel et vendre des reproducteurs.

\section{Quels sont les avantages et inconvénients d'avoir une telle exploita- tion en milieu périurbain?}

Les débouchés variés sont un réel avantage. Je produis des animaux vivants et d'autres qui partent en boucherie. Pour les porcs gascons, je vend quelques reproducteurs mais je fais surtout des porcelets qui seront en partie transformés en cochons de lait et en partie vendus à des engraisseurs, car c'est une viande très prisée et de grande qualité gustative ; pour les moutons landais, je fait quelques agneaux de lait mais c'est surtout les jeunes agnelles qui sont vendues pour aller recréer les cheptels; enfin, les poules gasconnes sont en partie vendues vivantes à des particuliers pour faire des œufs, mais je commercialise surtout des œufs.

Nous sommes à côté d'un bassin de population qui représente 800000 personnes, donc je ne peux pas dire que la commercialisation soit très compliquée, j'ai plus de demande que de production. Je ne fonctionne qu'en vente directe, de temps en temps en AMAP. Le développement des achats par particuliers se développe ; il y a des gens avec un peu de terrain qui prennent 
quelques brebis pour entretenir leurs parcelles, ou quelques poules, ce qui permet de diffuser ces races. L'ensemble du domaine est certifié agriculture biologique, et ça a été déterminant pour valoriser un produit haut de gamme et de qualité.

Mais il y a quelques inconvénients : nous payons aujourd'hui la désertification des campagnes d'il y a 40 ans, quand il n'y avait plus d'éleveurs et que les terres ont été récupérées pour construire de l'habitat ou des infrastructures, ou bien rachetées par des agriculteurs industriels; il y a donc peu d'élevages dans nos zones, et tous les abattoirs de proximité ont fermé ce qui nous pousse à faire des kilomètres pour faire abattre nos animaux. Cela avec tous les problèmes occasionnés par le stress, les déplacements, et l'incertitude quand à savoir si les abattoirs font correctement leur travail.

Avec l'expansion urbaine et la densification, la ville se rapproche, avec des populations qui viennent à proximité des élevages avec beaucoup d'idées reçues. Il y a une part d'éducation et d'explication à faire. Enfin, il y a le problème des maladies qui se diffusent avec les mouvements des populations ; au niveau vétérinaire, ont doit pouvoir protéger les habitants des maladies, mais aussi nos élevages des gens qui seraient involontairement porteurs. C'est une vigilance lourde, avec des procédures coûteuses et compliquées, qui risquent d'engendrer des coûts de production trop importants qui vont nécessairement se répercuter sur le prix de vente (à la base abordable grâce à la proximité des élevages avec les points de vente). Ceci pose la question de la survie des élevages à petite échelle sur ces zones périurbaines mais qui vont devenir inévitablement urbaines dans les années à venir. 